Supporting Information for

\title{
Modulating Magnetism in Ferroelectric Polymer-Gated Perovskite Manganite Films with Moderate Gate Pulse Chains
}

Hon Fai Wong ${ }^{1}$, Sheung Mei $\mathrm{Ng}^{1}$, Wen Zhang ${ }^{2,3}$, Yu Kuai Liul, Ping Kwan Johnny Wong',4, Chi Sin Tang ${ }^{3}$, Ka Kin Lam $^{1}$, Xu Wen Zhao , Zhen Gong Meng ${ }^{5}$, Lin Feng Feil, Wang Fai Cheng ${ }^{1}$, Danny von Nordheim ${ }^{6}$, Wai Yeung Wong ${ }^{7}$, Zong Rong Wang ${ }^{8}$, Bernd Ploss ${ }^{6}$, Ji-Yan Dail, Chee Leung Mak' ${ }^{1}$, Andrew Thye Shen Wee 3,4 , Chi Wah Leung ${ }^{1 *}$

1 Department of Applied Physics, The Hong Kong Polytechnic University, Hung Hom, Kowloon, Hong Kong, China

2 School of Electronics and Information and School of Microelectronics, Northwestern Polytechnical University, 127 West Youyi Road, Xi'an, Shaanxi, 710072, China

3 Department of Physics, National University of Singapore, 2 Science Drive 3, Singapore 117542, Singapore

4 Centre for Advanced 2D Materials and Graphene Research Centre, National University of Singapore, 6 Science Drive 2, Singapore 117546, Singapore

5 College of Chemistry and Environmental Engineering, Shenzhen University, Shenzhen 518060, China

6 Department of SciTec, University of Applied Sciences Jena, Carl-Zeiss-Promenade 2, 07743 Jena, Germany

7 Department of Applied Biology and Chemical Technology, The Hong Kong Polytechnic University, Hung Hom, Hong Kong, China

8 State Key Lab of Silicon Materials, School of Materials Science and Engineering, Zhejiang University, Hangzhou, China

* dennis.leung@polyu.edu.hk 


\section{Structural and ferroelectric characterization}

The structural properties of LSMO films grown on STO (001) substrates were previously studied by X-ray diffraction, which indicated highly-textured growth. ${ }^{1}$ Figure S1 (a) shows high-resolution XRD spectra of LSMO film before and after removing the P(VDF-TrFE) layer. The LSMO (002) peak in $2 \theta$ and its corresponding $\omega$-scan show minimal changes. Meanwhile, Kiessig fringes in X-ray reflectivity (XRR) measurement (Figure S1 (b)) indicates a film thickness of $7.5 \mathrm{~nm}$ with surface roughness $\sim 0.43 \mathrm{~nm}$, consistent with the results obtained by atomic force microscopy (AFM) $(0.38 \mathrm{~nm}$, inset of Figure $\mathrm{S} 1$ (b)). The AFM image also exhibits an atomically flat LSMO surface with clear terraces of step height $\sim 0.38 \mathrm{~nm}$, consistent with the unit-cell height of LSMO along the (001) cleavage plane. Such terraces were due to the formation of $\mathrm{TiO}_{2}$-terminated STO surfaces, which was obtained by DI water treatment of the substrates before LSMO deposition. We also note that our previous study has established the epitaxial nature of the LSMO prepared under the listed conditions. ${ }^{1}$

XRD scan of P(VDF-TrFE) on LSMO film (inset, Figure S1 (c)) shows the peak for (200) and (110) planes of $\mathrm{P}(\mathrm{VDF}-\mathrm{TrFE})$ at $\sim 19^{\circ}$, after annealing the sample in air at $120^{\circ} \mathrm{C}$ for two hours, implying the presence of ferroelectric $\beta$ phase. ${ }^{2}$ Ferroelectric hysterics loop of P(VDF-TrFE) film (Figure S1 (c)) measured at $100 \mathrm{kHz}$ and $300 \mathrm{~K}$ exhibits a remnant polarization of $10 \mu \mathrm{C}$ $\mathrm{cm}^{-2}$, coercive voltage of $15 \mathrm{~V}$ and saturation voltage of $40 \mathrm{~V}$.

Figure S1 (d) shows high-resolution cross-section TEM image of $25 \mathrm{~nm}$ thick LSMO sample on NSTO (001) substrate, after $36 \mathrm{k}+V_{g}$ pulse cycles and with the P(VDF-TrFE) removed by organic solvent. The TEM image shows the sharp interface between LSMO and NSTO, and no apparent misfit dislocation on the LSMO surface (previously in contact with P(VDF-TrFE)). Figure S1(d) show the [010] zone axis of selected area electron diffraction (SAED) patterns of the LSMO/NSTO. All diffraction spots belong to LSMO/NSTO. No diffraction ring and splitting of diffraction spots are observed, indicating an epitaxial growth of the LSMO films on NSTO substrate and absence of additional phases or crystal forms after gating with P(VDF-TrFE). 

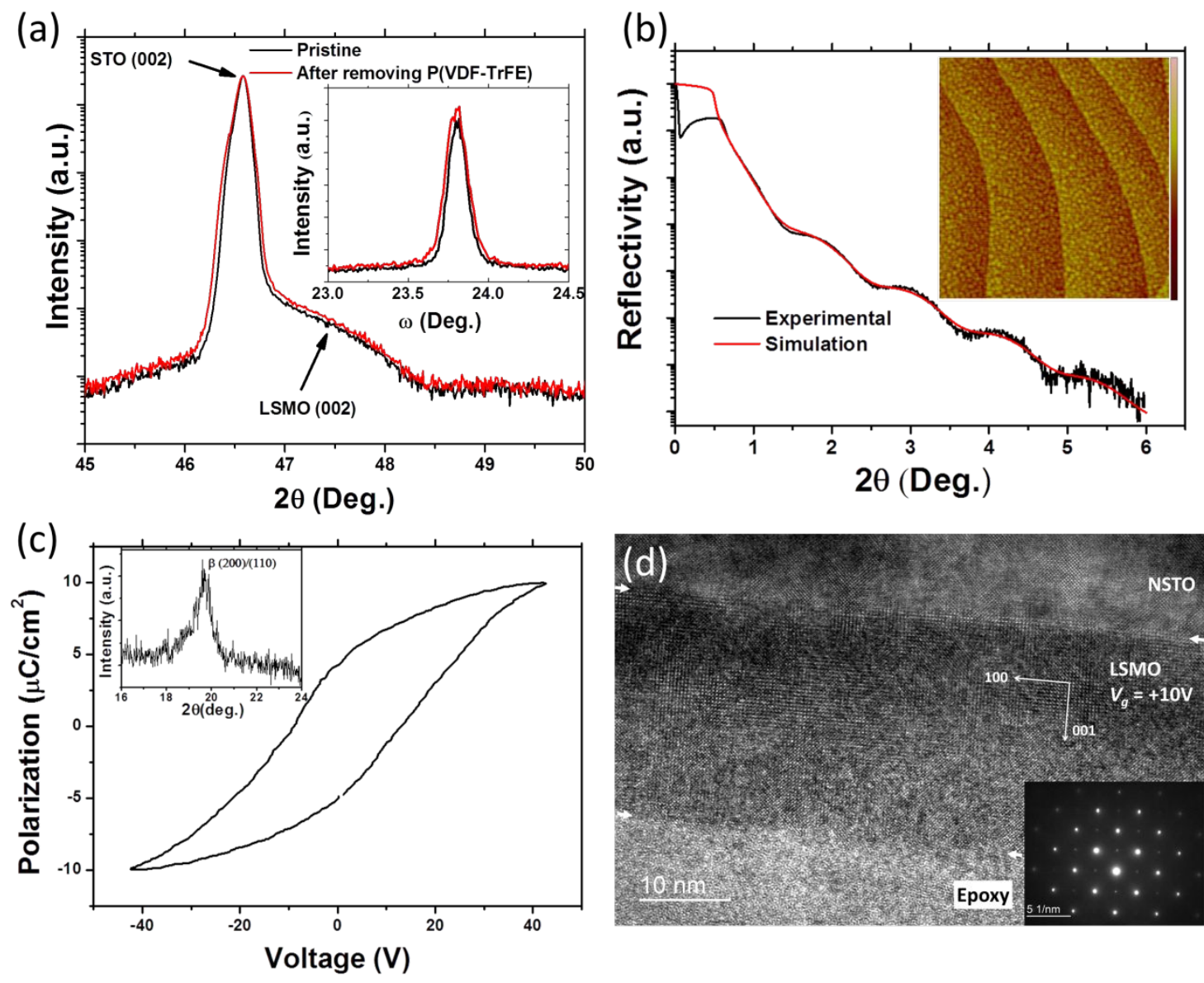

Figure S1. (a) XRD spectrum of 7.5 nm-thick LSMO films grown on $\mathrm{TiO}_{2}$-terminated STO (001) substrate with pristine state and after removing P(VDF-TrFE), with inset showing the $\omega$-scan of the LSMO (002) peak. (b) XRR measurement of $7.5 \mathrm{~nm}$-LSMO. Inset shows the AFM scan of the LSMO film (scan area $=1 \mu \mathrm{m}^{2}, \mathrm{z}=4$ $\mathrm{nm}$ ). (c) Polarization hysteresis loop of $\mathrm{P}(\mathrm{VDF}-\mathrm{TrFE})$ prepared on glass substrate, with the inset showing the XRD $\theta-2 \theta$ scan profile of P(VDF-TrFE) on LSMO film surface. (d) Cross-sectional TEM image of the $25 \mathrm{~nm}$ thick LSMO/NSTO. The inset is the SAED patterns of LSMO/NSTO. 


\section{Characterization of field-effect devices with $V_{g}$ pulses}
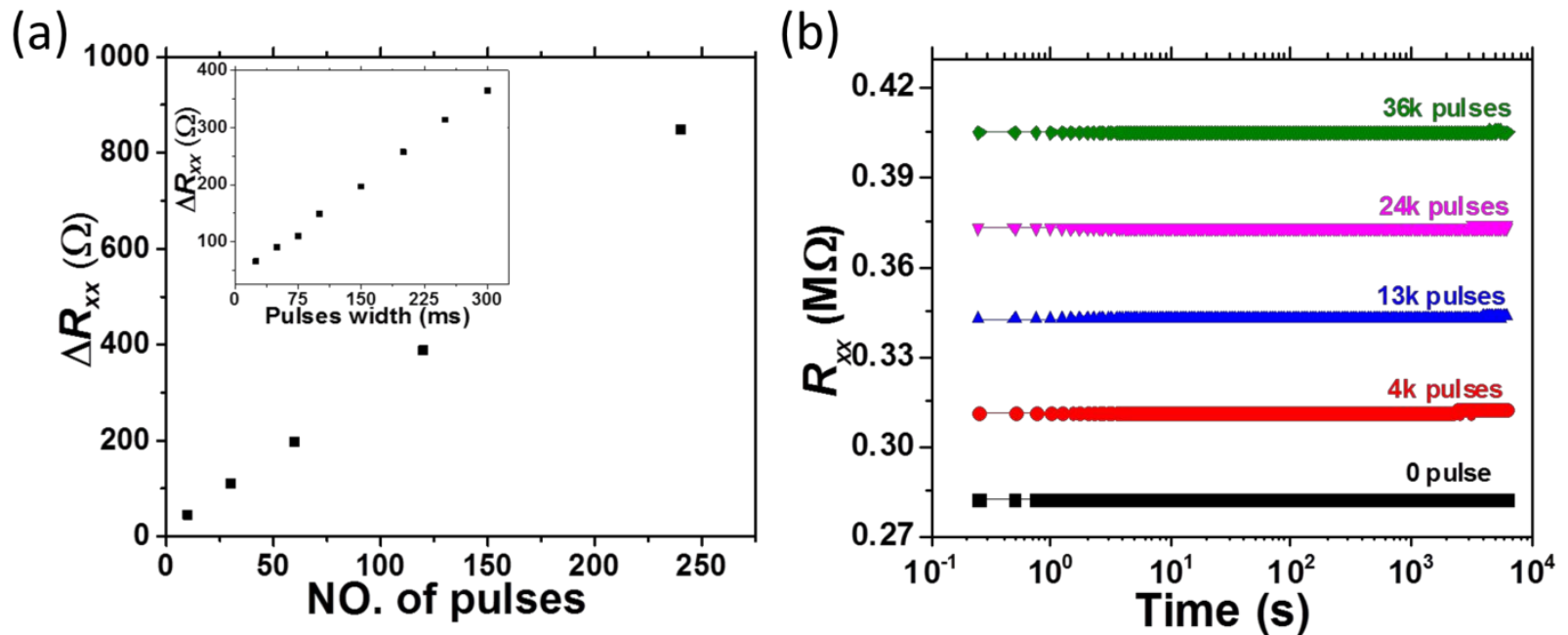

Figure S2. (a) LSMO channel resistance change $\left(\Delta R_{x x}\right)$ at $300 \mathrm{~K}$ as a function of $\mathrm{V}_{\mathrm{g}}$ pulse counts (with constant amplitude $10 \mathrm{~V}$ and width of $300 \mathrm{~ms}$, main figure), and as a function of pulse width (with constant amplitude $10 \mathrm{~V}$ and 60 pulse counts, inset). (b) shows the time dependence of $R_{x x}$ after applying different number of $+V_{g}$ pulses ( 0 to $36 \mathrm{k}$ cycles).

A field-effect transistor (FET)-like device structure with LSMO channel and P(VDF-TrFE) gate dielectric was used in this study, the schematic of which is shown in Figure 1(a) of main text. In the figure, $R_{x x}$ is the four-point longitudinal resistance of LSMO channel, and $V_{g}$ is the $\mathrm{P}(\mathrm{VDF}-\mathrm{TrFE})$ gate potential with reference to the LSMO channel (drain). Unless otherwise stated, $V_{g}$ magnitude, pulse width and pulse cycle used in the work are $10 \mathrm{~V}, 300 \mathrm{~ms}$ and $500 \mathrm{~ms}$ respectively. In Figure S2, the transport properties of LSMO channel at $300 \mathrm{~K}$ are shown, after undergoing various $V_{g}$ pulse cycles. Here $\Delta R_{x x} / R_{o}(\%)$ and $\Delta R_{x x}$ are defined as $\left.\left(R_{x x}(n)-R_{o}\right) / R_{o}\right)$ and $R_{x x}(n)-R_{o}$ respectively, where $n$ is the number of pulses applied to the channel and $R_{o}=R_{x x}(n$ $=0)$ refers to the channel resistance at the start of the measurement before $V_{g}$ was applied. The results indicate that $\Delta R_{x x}$ correlates positively with the number of pulses applied (main figure) and the pulse width (inset). As shown in Figure S2(b), $R_{x x}$ increases progressively from $0.28 \mathrm{M} \Omega$ to $0.41 \mathrm{M} \Omega$ through the application of $36 \mathrm{k}$ pulses. $R_{x x}$ remains stable when $+V_{g}$ pulsing is stopped. The gradual change and stability of $R_{x x}$ therefore highlights the possibility of achieving multiple resistance states in the LSMO channel by applying moderate pulses. 


\section{Reversible modulation of LSMO channel properties}

Positive and negative $V_{g}$ pulse chains were applied to evaluate the reversibility of LSMO channel properties $\left(R_{x x}, T_{C}\right.$ and MR), and the results are depicted in Figure S3. Different stages in the figure ( $x$-axis) correspond to states of the LSMO channel after the application of certain $+/-V_{g}$ pulses (Figure S3(a)). It should be noted that different counts of $+V_{g}$ and $-V_{g}$ pulses were needed to increase or decrease $R_{x x}$ by approximately same amount; such asymmetry in switching is clearly demonstrated in Figure 3 in the main text.

At the start of the experiment (Stage 1), $R_{x x}$ of the pristine LSMO channel is $0.14 \mathrm{M} \Omega$ at 300 $\mathrm{K}$, and $T_{C}$ of the LSMO channel is $303 \mathrm{~K}$, while the MR at $20 \mathrm{~K}$ is $-0.25 \%$. With the application of $+V_{g}$ pulse cycles (Stage 2), $R_{x x}$ becomes $0.41 \mathrm{M} \Omega$ at $300 \mathrm{~K}$, accompanied by a decrease in $T_{C}$ to $263 \mathrm{~K}$. The increase in $R_{x x}$ at $300 \mathrm{~K}$, and the corresponding decrease of $T_{C}$ in the LSMO channel can be explained by the depletion of hole carriers, which is consistent with the increase of magnetic scattering during low-temperature MR measurement (-0.90\%). With the subsequent application of $-V_{g}$ pulse cycles that resets the $R_{x x}$ at $300 \mathrm{~K}$ back to $0.28 \mathrm{M} \Omega$ (Stage 3), $T_{C}$ and $\mathrm{MR}$ at $20 \mathrm{~K}$ are changed to $280 \mathrm{~K}$ and $-0.54 \%$ respectively. This indicates that the gate-induced accumulation of hole carriers in LSMO channel can be achieved by the application of $-V_{g}$ pulse cycles. In Stage 4 , the $T_{C}$ and MR at $20 \mathrm{~K}$ become $265 \mathrm{~K}$ and $-1 \%$, as $R_{x x}$ at $300 \mathrm{~K}$ is reset to 0.41 $\mathrm{M} \Omega$ once again upon the application of $+V_{g}$ cycles. Similarly, the application of $-V_{g}$ chains brings the $R_{x x}$ at $300 \mathrm{~K}$ to $0.28 \mathrm{M} \Omega$, accompanied by the rise in $T_{C}$ and drop of MR magnitude at $20 \mathrm{~K}$ (Stage 5). Similar tunability of $T_{C}$ and low temperature-magnetotransport properties were observed for more than 10 LSMO devices. Our previous work showed that two non-volatile resistance states could be achieved by applying only $60+V_{g}$ pulse cycles and they were reversible by applying similar number of pulses, but with limited resistance change. ${ }^{1}$ 


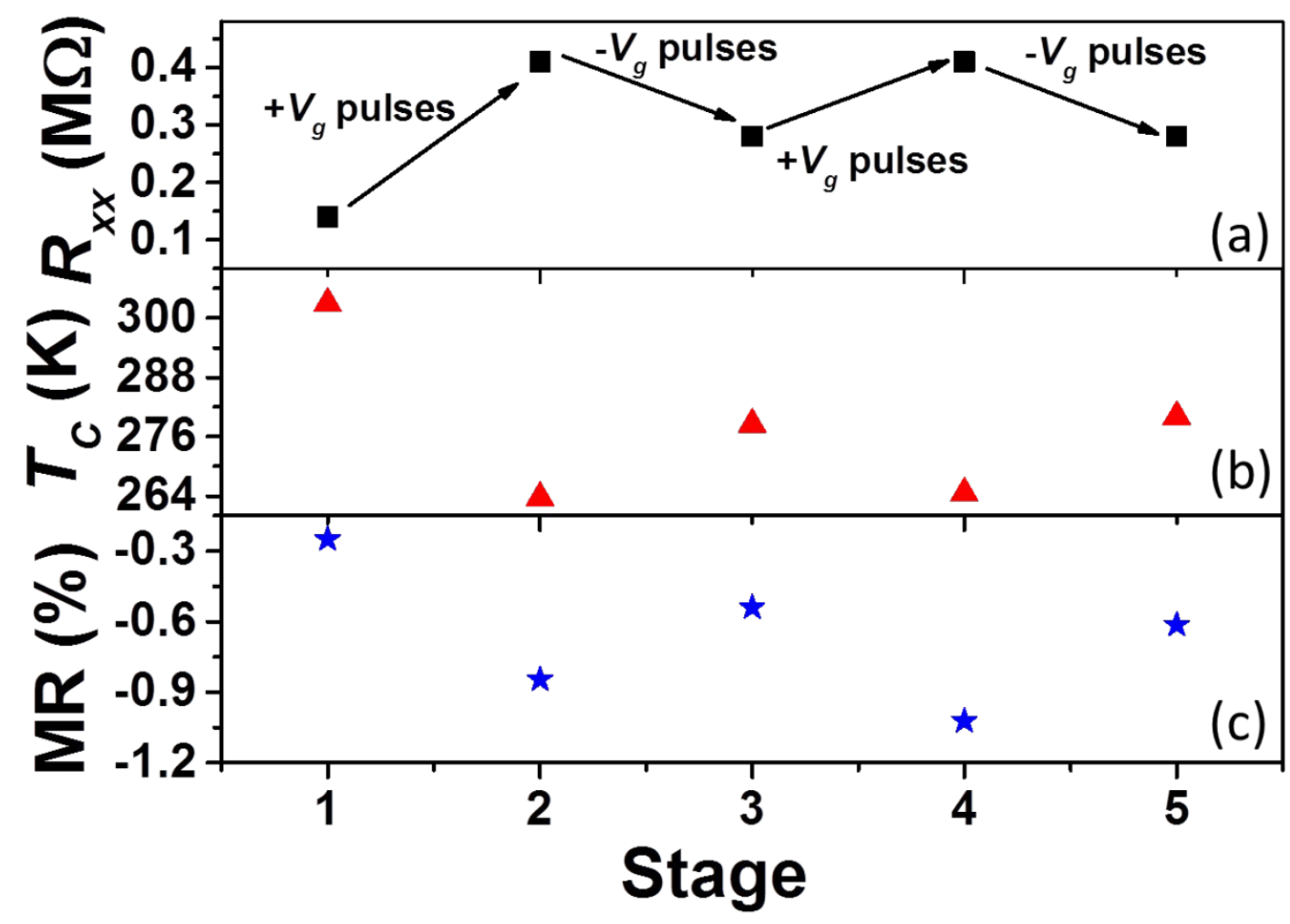

Figure S3. Reversibility of LSMO channel resistance at $300 \mathrm{~K}$ (a), $T_{C}$ of LSMO (b), and MR of LSMO channel at 20 $\mathrm{K}(\mathrm{c})$, through the application of positive and negative $V_{g}$ pulse chains.

\section{Modulation of $\boldsymbol{R}_{x x}$ under oxygen and vacuum ambient conditions}
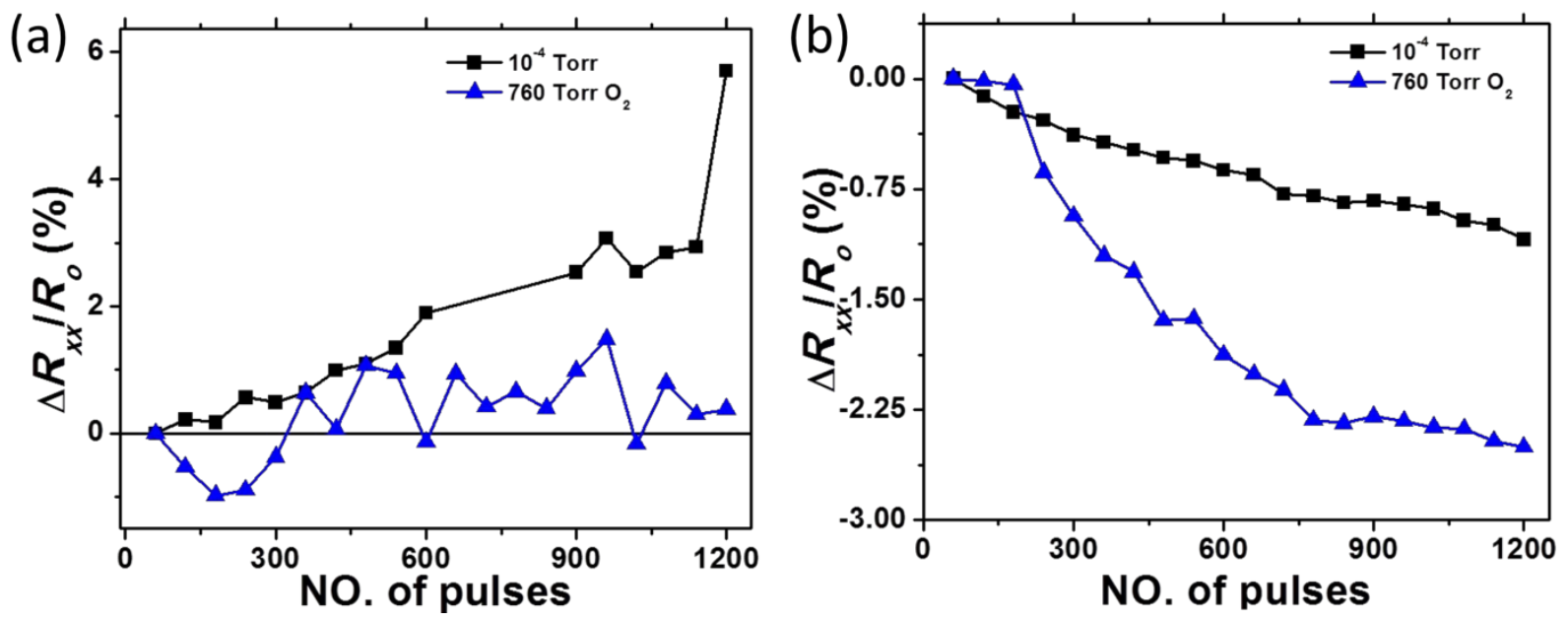

Figure S4. $\Delta R_{x x} / R_{o}$ variation with pulse counts under $+V_{g}$ (a) and $-V_{g}(\mathrm{~b})$. Measurements were conducted either in vacuum (squares) or in oxygen (triangles) environment. Results were recorded at $300 \mathrm{~K}$.

Figure S4 shows the variation of $\Delta R_{x x} / R_{o}$ of LSMO channel with $+/-V_{g}$ pulses under different environment. For $+V_{g}$ pulses (Figure $\mathrm{S} 4$ (a)), while fluctuation in $\Delta R_{x x}$ change is 
observed when the channel is switched in $\mathrm{O}_{2}\left(99.7 \%\right.$ purity) environment, a gradual $R_{x x}$ increase is obtained when the sample is pulsed in vacuum. One can postulate that the vacuum environment can accelerate the LSMO resistance change, as a consequence of the efficient removal of oxygen (i.e. creation of $V_{o}$ ) in vacuum. For $-V_{g}$ pulses (Figure S4(b)), although a $R_{x x}$ drop is observed in both vacuum and oxygen environment, the effect is doubled in the presence of oxygen. This suggests the incorporation of oxygen into LSMO channel which helps to annihilate $V_{o}$.

\section{Supplementary Information References}

(1) Wong, H. F.; Ng, S. M.; Cheng, W. F.; Liu, Y.; Chen, X.; von Nordheim, D.; Mak, C. L.; Dai, J.; Ploss, B.; Leung, C. W. Enhanced Tunability of Electrical and Magnetic Properties in (La,Sr) $\mathrm{MnO}_{3}$ Thin Films via Field-Assisted Oxygen Vacancy Modulation. Solid-State Electron. 2017, 138, 56-61.

(2) Hu, W. J.; Juo, D. M.; You, L.; Wang, J.; Chen, Y. C.; Chu, Y. H.; Wu, T. Universal Ferroelectric Switching Dynamics of Vinylidene Fluoride-trifluoroethylene Copolymer Films. Sci. Rep. 2014, 4, 4772. 